\title{
Scale-free Network Models With Parameters
}

\author{
Fei $\mathrm{Ma}^{1, \mathrm{a}}$, Jing $\mathrm{Su}^{2, \mathrm{a}}$, Bing $\mathrm{Yao}^{3, \mathrm{a}^{*}}$, Ming $\mathrm{Yao}^{4, \mathrm{~b}}$ \\ ${ }^{a}$ College of Mathematics and Statistics, Northwest Normal University, \\ Lanzhou, Gansu CHINA 730070 \\ bDepartment of Information Processand Control Engineering, Lanzhou \\ Petrochemical College of Vocational Technology \\ Lanzhou, Gansu CHINA 730070 \\ *yybb@163.com
}

Keywords: scale-free network; dynamical evolution; dynamic equation; small-world effect; preferential attachment.

\begin{abstract}
Based on typical scale-free network models, we set up the partial differential equation satisfied more general dynamic systems, and then we not only find another important topological property of scale-free networks, but also discuss the real background meaning of every function in the dynamical systems. Meanwhile we generalize the BA-network-model growth way ("star-graph-growth-way"). Starting from a more general situation, we establish a network model containing "star-graph-growth-way" and "triangle-growth-way". By analysis, this model is not only scale-free but also small-wall. Finally, distinguish the connect between the scope of the power law parameters.
\end{abstract}

\section{Introduction}

In the past twenty years we have seen a peak in the research of a wide range of complex systems which can be described in terms of networks--vertices connected together by edges. Small-world effect (any two vertices in the system can be connected by relatively short paths and local clustering characterizes the tendency of groups of vertices to be all connected to each other) and Scale-free property (vertices degree distribution follow a power-law) are common in most real-life networks(Ref. [6], [7], [9], [18], [21], [22], [23], [29], [30], [31]), transportation systems or social and economic networks and so on. Complex networks with these two characteristics are called small-world scale-free networks. The time sees three steps on studying complex systems and networks. The first step: In the 1950s, based on researching the classic the graph theory, Erdös and Rényi (Ref. [15]), as the pioneers of this investigation, came up with the stochastic network model (ER-model) to describe complex network. The second step: Watts and Strogatz (Ref. [2], [4]) put forward the small-world model (WS-model) in 1998. The third step: By researching the topological structure of WWW, Barabasi and Albert (Ref. [3]) presented firstly the scale-free model (BA-model) $P(k) \approx k-\gamma$ in their article published in 1999, where $\gamma$ is called as scale-free parameter and its value range follows $2<\gamma<3$ at present (Ref. [20]). It is so clear for us that every step has itself focus. The researching way has been pushed from "regualtion and stochastical" to "the complex".

ER-model has smaller average path length no higher clustering coefficient. WS-model has the same as the degree distribution of ER-model, poisson distribution. The clustering coefficient of BA-model presented drops drastically from a very high value to a smaller one (even 0) when increasing time $t$ from 0 to $\infty$. In a words, three networks models mentioned above can not describe better numbers of real-life networks having small-world effect and scale-free property. It is an urge research project how to design some better models. Taking three representative models into account, researchers generate abundant various models, each one is structured with individual angle, which have scale-free property no small-world effect like stochastic models BA scale-free two-way evolution network models (Ref. [29]), an evolving model for scale-free collaboration networks (Ref. [31]), a dynamic scale-free network model (Ref. [23]), etc. Apparently, these new vertices of each 
model are added into networks in isolation in this dynamical process. The result leads to it with lower cluster coefficient. For obtaining higher one, some studier build some deterministic models like Apollonian models (Ref. [8], [13]), Farey graph models (Ref. [32]), Sierpinski models (Ref. [33]) and so on. These models have small-world effect but no randomly generating. The dynamical evolution of most real-life networks is random and free. So them are not ideal ones. The writer (Ref. [30]) says a stochastic model named group growing model based small-world scale-free network which has these two characteristics.

In order to making models built having small-world effect and scale-free property, probers come up with two ways, the direct and the indirect.

Direct way. Make models having these two characteristics in the dynamical evolution process.

Indirect way. After the models have been built, make models having these two characteristics by taking many operations. The good sample is WS-model is generated on the base of ER-model by removing some edges and adding some new edges with one certain probability. Such better models do not win more attentions.

Firstly, we, in this paper, establish a dynamical equation relatively with the dynamical evolution process of complex systems and networks. Secondly, we design one class tuned parameters small-world scale-free network Models by improving the simple entering way of adding some new isolate vertices into networks. Some useful definitions (Ref. [1], [5]) are mentioned below.

Average Path Length (APL). The distance between two vertices is the least number of edges to get from one vertex to the other. The average path length is the smallest number of links connecting a pair of vertices, averaged over all pairs of vertices.

Clustering Coefficient. The clustering coefficient $C_{i}$ of a vertex $\mathrm{i}$ is the ratio of the total number ei of existing edges between all $k_{i}$ it nearest neighborhoods and the number $k_{i}\left(k_{i}-1\right) / 2$ of all possible edges between them, ie $C_{i}=2 e_{i} / k_{i}\left(k_{i}-1\right)$. The cluster coefficient $c$ of the whole network is the average value of all individual $C_{i} \mathrm{~s},\langle C\rangle=\Sigma C_{\mathrm{i}} / n_{v}(t)$.

\section{One dynamical partial differential equation}

Making some descriptions and states for the dynamical evolution process, which contains some new vertices entered continuously, some new edges generated continuously, some old vertices removed continuously, also, some old edges removed continuously and much interference from the outside at each time step, of both complex networks and complex system. Researchers not only apply theoretical analysis, but also put to use simulation by computer, there exists lots of similarity about these under all almost evolutions happening under this given preferential attachment mechanism. As time goes on, the scope of the dynamical complex network become more and more huger by these discussions. In the other word, the number vertices and edges increase and its spacial structure will become more complex. Nothing will always grow unceasingly. Undergoing after an extended period, network itself will tend to a stable or decay. Combining the real background meanings and theoretical analysis, we will make a sample depiction about the dynamical evolution way of complex systems and complex networks by introducing 5 characteristic functions for a network model $N(t)$.

(1) The entering vertices function (preferential attachment function) $f^{*}(t)=f\left(a p_{1}(t) m, t, k_{i}(t)\right.$, $\left.\Sigma \prod_{l j}\left(k_{i}\right)\right)$ indicates that there are $a$ new vertices entered in the network $N(t-1)$ at time step $t$. The new vertex $j_{a}$ will devote $p_{l}(t) m\left(0<p_{l}(t)<1\right)$ edges to $N(t)$ by connecting with some old vertices in $N(t-1)$. The probability which the degree $k_{i}(t)$ vertex obtains new links obeys the preferential attachment probability $\prod_{l j}\left(k_{i}\right)$, where $\Sigma \prod_{l j}\left(k_{i}\right)$ stands for all kinds of probability co-existing (the most important is preferential attachment probability).

(2) The removing vertices function $g^{*}(t)$. After network goes through the quickly increasing process, $g^{*}(t)=g\left(p_{2}(t) b, t, k_{i}(t), \Sigma \prod_{l j}\left(k_{i}\right)\right)$ shows that there are $\left(p_{2}(t) b\left(0<p_{2}(t)<1\right)\right.$ old vertices removed from the network $N(t-1)$ at time step $t$. An old vertex $j_{b}$ will be removed with the probability $\prod_{l j}\left(k_{i}\right)$ (the opposite-preferential attachment probability). 
(3) The adding edges function. $h^{*}(t)=h\left(p_{3}(t) r, t, k_{i}(t), \Sigma \prod_{3 j}\left(k_{i}\right)\right)$ suggests there will be $p_{3}(t) r$ $\left(0<p_{3}(t)<1\right)$ "new"edges added among a few pairs of vertices which are not linked in the network $N(t-1)$ at time step $t$. The two vertices linked by one new edge $j_{r}$ will be selected with the preferential attachment probability $\prod_{3 j}\left(k_{i}\right)$.

(4) The canceling edges function. $z^{*}(t)=z\left(p_{4}(t) s r, t, k_{i}(t), \Sigma \prod_{4 j}\left(k_{i}\right)\right)$ suggests there will be $p_{4}(t) s$ $\left(0<p_{4}(t)<1\right)$ "new"edges added among a few pairs of vertices which are not linked in the network $N(t-1)$ at time step $t$. The two vertices linked by one new edge $j_{s}$ will be selected with the preferential attachment probability $\prod_{4 j}\left(k_{i}\right)$.

(5) The external disturbance function. $\varphi(t)$ hints the process of networks evolution will endure unavoidable influence from the outside world.

It is obvious for us that both the internal function from its internal factor (adding edges, removing vertices, canceling edges) and the external factor (entering vertices, disturbance) will make the dynamic evolution of the network become most harmonious.

We may suppose the dynamic evolution of a connected original network $N(0)$ having $m_{0}$ vertices is continual and then can set up a corresponding partial differential equation to explain this dynamical system, according to the independence of events. There is a corresponding partial differential equation for a degree $k_{i}(t)$ vertex in the network $N(t)$ at time step $t$ shown as

$\frac{\partial k_{i}(t)}{\partial t}=f^{*}(t)+g^{*}(t)+h^{*}(t)+z^{*}(t)+\varphi(t)$

We are permitted to suppose the equation (1) having a solution

$k_{i}(t)=\theta\left(t, t_{i}, \alpha_{1}, \alpha_{2}, \cdots, \alpha_{r}\right)$

These parameters $\alpha_{i}(i=1,2, \ldots, r)$ in (2) is irrelative with $t$ and $t_{i}$, moving forward

$P\left(k_{i}(t)<k\right)=P\left(t_{i}>\theta^{-1}\left(k_{i}(t), t, \beta_{1}, \beta_{2}, \cdots, \beta_{r}\right)\right)$

As the same result, these parameters $\beta_{i}(i=1,2, \ldots, r)$ in (3) is also irrelative with $t$ and $t_{i}$. Finally, we acquire the arisen probability of vertices of degree $k$ in $N(t)$.

\section{Dynamic models in communication networks}

Any real complex network can not work by itself. In the dynamical evolution process, it will be influence by some factors from itself internal and the outside. There are many influence helpful for it, others will be bad. For this network growth process at present, learners almost apply the method that new vertices will be entered randomly into network and then link some old vertices by the preferential attachment probability. Obviously, these research is limit. Try to imagine, in real society, not only are some vertices entered into network, but also are many sample graph entered into it. At the same time, each new vertex can link some old vertices by different attachment ways. For example, in a real communication network, a classical scale-free network model, named collaboration network, is made by Zhang (Ref. [31]) in a concrete condition that a new person will also make friends with these people having highly connections in each other. The ideal model is rare in life. From the real background meaning, we have established a scale-free network model, named two different preferential attachment probability co-exist network, in this real suppose that a part of people like making friends with these people having highly connections in each other; parts of people prefer to communicate with those persons having more plenty friends (named commonly as social strong hands); others who have even no a clear goal and a plain understanding about the social network make randomly friends with some people. There is a general phenomenon that some people like making new friends alone, however another will introduce their old friends to those new friends just known soon really existence. Considering the real background, we bring about 3 classes tuned parameters small-world scale-free network models in this paper. There will be some detailed discussions talked below. 


\section{A New models in communication networks}

The constructing process of $N(t)$ is in the way: the original network $N(0)$ is a connected simple graph having $n_{0} \geq 2$ vertices and $m_{0} \geq 1$ edges. From time step $t \geq 1$, these two operations below will be carry out.

Growth. At time step $t$, there is a group of $2 a$ new vertices entered into the network $N(t-1)$.

The Preferential Attachment Operation. This group of vertices are made up two classes different vertices. The number of the first part is $2 \alpha a$, another is $2(1-\alpha) a$. Each vertex of the first part links randomly $m\left(\leq m_{0}\right)$ old vertices in $N(t-1)$ by using the preferential attachment probability $k_{i} / \Sigma k_{j}$. The second part contains two classes different vertices, $A$-class and $B$-class. Any two vertices of the second part will generate a new edge by link each other. The operation leads to $(1-\alpha) a$ new edges. Analogously, the two classes vertices, $A_{1}$-class and $A_{2}$-class, belong to the $A$-class. The two vertices of a new edge of the $A_{1}$-class, including $2 \mu \beta(1-\alpha) a$ vertices, will simultaneously link randomly $m-1\left(\leq m_{0}\right)$ old vertices in $N(t-1)$ by using the preferential attachment probability $k_{i} / \Sigma k_{j} . m_{3}$ edges incident with each end of a new edge of the $A_{2}$-class, including $2(1-\mu) \beta(1-\alpha) a$ vertices, will simultaneously link randomly any $m_{3}\left(\leq m \leq m_{0}\right)$ old vertices in $N(\mathrm{t}-1)$ by using the preferential attachment probability $k_{i} / \Sigma k_{j}$. Another end of the new edge of the $A_{2}$-class will link randomly $m-1-m_{3}$ diverse old vertices in $N(t-1)$ by using the preferential attachment probability $k_{i} / \Sigma k_{j}$. Every end of a new edge of the $B$-class, including $2(1-\beta)(1-\alpha) a$ vertices, will link randomly $m-1\left(\leq m_{0}\right)$ diverse old vertices in $N(t-1)$ by using the preferential attachment probability $k_{i} / \Sigma k_{j}$. This growth process may circularly go on until an expected model is captured. Obviously, $m_{3}$ edges incident with each end of a new edge of the $A_{2}$-class links randomly $m_{3}\left(\leq m \leq m_{0}\right)$ diverse old vertices in $N(t-1)$ will bring $m_{3}$ triangles (i.e. $\left.K_{3}\right)$ which shows a man having a old friend make friends with $m-1$ unknown persons and then introduce his old friend to $m_{3}$ of those new friends just known soon by the way. After $t$ times, the network model $N(t)$ has $n_{v}(t)=n_{0}+2 a t$ vertices and $e_{v}(t)=m_{0}+[2 a m-(1-\alpha) a] t$ edges.

Assuming that $k_{i}$ is a continuous real variable. According to its dynamical evolution mechanism.

Case A1. A degree $k_{i}$ vertex only links simultaneously $m_{3}$ edges incident with each end of any new edge of the $A_{2}$-class, so the entering vertices function is

$$
f_{3.1}^{*}(t)=2 \alpha a m \frac{k_{i}}{2 m_{0}+2[2 a m-(1-\alpha) a] t}+\frac{\left[\mu \beta(1-\alpha) a \cdot(m-1)^{2}\right] k_{i}}{2 m_{0}+2[2 a m-(1-\alpha) a] t}+\frac{[(1-\beta)(1-\alpha) a \cdot(m-1)] k_{i}}{2 m_{0}+2[2 a m-(1-\alpha) a] t}+\frac{\left[(1-\mu) \beta(1-\alpha) a \cdot m_{3}^{2}\right] k_{i}}{2 m_{0}+2[2 a m-(1-\alpha) a] t}
$$

Case A2. A degree $k_{i}$ vertex only links simultaneously $m-1-m_{3}$ edges incident with each end of any new edge of the $A_{2}$-class, which gives the entering vertices function as

$$
f_{32}^{*}(t)=2 \alpha a m \frac{k_{i}}{2 m_{0}+2[2 a m-(1-\alpha) a] t}+\frac{\left[\mu \beta(1-\alpha) a \cdot(m-1)^{2}\right] k_{i}}{2 m_{0}+2[2 a m-(1-\alpha) a] t}+\frac{[(1-\beta)(1-\alpha) a \cdot(m-1)] k_{i}}{2 m_{0}+2[2 a m-(1-\alpha) a] t}+\frac{\left[(1-\mu) \beta(1-\alpha) a \cdot\left(m-m_{3}-1\right)\right] k_{i}}{2 m_{0}+2[2 a m-(1-\alpha) a] t}
$$

Case A3. A degree $k_{i}$ vertex not only links $m-1-m_{3}$ edges incident with each end of any new edge of one part, including $(1-u)(1-\mu) \beta(1-\alpha) a$ new edges, of the $A_{2}$-class, but also links $m_{3}$ edges incident with each end of any new edge of another part, including $\mathrm{u}(1-\mu) \beta(1-\alpha) a$ new edges, of the $A_{2}$-class. Then we have the entering vertices function

$$
\begin{aligned}
f_{33}^{*}(t)=2 \alpha a m & \frac{k_{i}}{2 m_{0}+2[2 a m-(1-\alpha) a] t}+\frac{\left[\mu \beta(1-\alpha) a \cdot(m-1)^{2}\right] k_{i}}{2 m_{0}+2[2 a m-(1-\alpha) a] t}+\frac{[(1-\beta)(1-\alpha) a \cdot(m-1)] k_{i}}{2 m_{0}+2[2 a m-(1-\alpha) a] t}+\frac{\left[u(1-\mu) \beta(1-\alpha) a \cdot m_{3}^{2}\right] k_{i}}{2 m_{0}+2[2 a m-(1-\alpha) a] t} \\
& +\frac{\left[(1-u)(1-\mu) \beta(1-\alpha) a \cdot\left(m-m_{3}-1\right)\right] k_{i}}{2 m_{0}+2[2 a m-(1-\alpha) a] t}
\end{aligned}
$$

We take others functions $g *(t)=h *(t)=z^{*}(t)=\varphi(t)=0$. Consequently, $k_{i}$ satisfies the dynamical equation

Case B1.

$$
\frac{\partial k_{i}(t)}{\partial t}=f_{3.1}^{*}
$$

Case B2.

$$
\frac{\partial k_{i}(t)}{\partial t}=f_{3.2}^{*}
$$


Case B3.

$$
\frac{\partial k_{i}(t)}{\partial t}=f_{33}^{*}
$$

These equations (4), (5) and (6) can can be written as

$$
\frac{\partial k_{i}(t)}{\partial t}=Q_{3, j} \frac{k_{i}}{t}
$$

with $j=1,2,3$, where

$$
Q_{3.1}=\frac{2 \alpha a \cdot m}{2[2 a m-(1-\alpha) a]}+\frac{(1-\alpha) a\left[\mu \beta(m-1)^{2}+(1-\beta)(m-1)\right]}{2[2 a m-(1-\alpha) a]}+\frac{(1-\alpha) a(1-\mu) \beta m_{3}^{2}}{2[2 a m-(1-\alpha) a]}
$$

and

$$
Q_{32}=\frac{2 \alpha a \cdot m}{2[2 a m-(1-\alpha) a]}+\frac{(1-\alpha) a\left[\mu \beta(m-1)^{2}+(1-\beta)(m-1)\right]}{2[2 a m-(1-\alpha) a]}+\frac{(1-\alpha) a(1-\mu) \beta\left(m-m_{3}-1\right)}{2[2 a m-(1-\alpha) a]}
$$

and

$$
Q_{33}=\frac{2 \alpha a \cdot m}{2[2 a m-(1-\alpha) a]}+\frac{(1-\alpha) a\left[\mu \beta(m-1)^{2}+(1-\beta)(m-1)\right]}{2[2 a m-(1-\alpha) a]}+\frac{(1-\alpha) a u(1-\mu) \beta m_{3}^{2}}{2[2 a m-(1-\alpha) a]}+\frac{(1-\alpha) a(1-u)(1-\mu) \beta\left(m-m_{3}-1\right)}{2[2 a m-(1-\alpha) a]}
$$

The solutions of these equations above (7) with $j=1,2,3$, when the initial condition that each vertex $i$ at its introduction has $k_{i}\left(t_{i}\right)=m$, are respectively as

$$
k_{i}(t)=m\left(\frac{t}{t_{i}}\right)^{Q,}
$$

and let $M_{3, j}(k)=\frac{m^{1 / Q_{,},}}{k^{1 / Q_{1}}}$ with $j=1,2,3$. According to $(8)$, the probability $P\left(k_{i}(t)<k\right)$ that one vertex has a degree $k_{i}(t)$ smaller than $k$ can be written as

$P\left(k_{i}(t)<k\right)=P\left(t_{i}>t M_{3, j}(k)\right)=1-P\left(t_{i} \leq t M_{3, j}(k)\right)$

with $j=1,2,3$. Suppose the density function $P\left(t_{i}\right)=1 /\left(m_{0}+t_{i}\right)$ for adding these new vertices at equal time interval into the network. Then we can compute

$$
P\left(t_{i} \leq t M_{3, j}(k)\right)=\int_{0}^{M_{i}(k)} P\left(t_{i}\right) d t=\frac{M_{3, j}(k)}{m_{0}+t_{i}}
$$

with $j=1,2,3$. Thereby, the degree distribution $P(k)$ for each case can be obtained

$$
P(k)=\frac{\partial P\left(k_{i}(t)<k\right)}{\partial k}=\frac{m^{1 / Q_{i}} t}{\left(m_{0}+t\right) Q_{3, j} k^{1 / Q_{,},+1}}
$$

with $j=1,2,3$. Under three cases Case B1, Case B2 and Case B3, as $t \rightarrow \infty$, predict asymptotically

$$
P(k) \approx \frac{m^{1 / Q_{2}}}{Q_{3, j}} \cdot \frac{1}{k^{1 / Q_{,}, 1}}
$$

with $j=1,2,3$. Clearly, they obey the BA-model $P(k) \approx k^{-\gamma}$.

\section{$B$ A new property}

We will calculate these equations (4), (5) and (6) by cumulation

$\sum\left|V\left(k_{i}, t\right)\right| \frac{\partial k_{i}(t)}{\partial t}$

where $\left|V\left(k_{i}, t\right)\right|$ stands for the number of vertices having degree $k_{i}$ at time step $t$. It is evident that this equation (12) attend to the constant values of Case B1, Case B2 and Case B3 as follows

$$
\begin{aligned}
& C_{3.1}=2 \alpha a m+(1-\alpha) a \mu \beta(m-1)^{2}+(1-\alpha) a\left[(1-\beta)(m-1)+(1-\mu) \beta m_{3}^{2}\right] \\
& C_{3.1}=2 \alpha a m+(1-\alpha) a \mu \beta(m-1)^{2}+(1-\alpha) a\left[(1-\beta)(m-1)+(1-\mu) \beta\left(m-m_{3}-1\right)\right] \\
& C_{3.1}=2 \alpha a m+(1-\alpha) a \mu \beta(m-1)^{2}+(1-\alpha) a\left\{(1-\beta)(m-1)_{3}^{2}+(1-\mu) \beta\left[u m_{3}^{2}+(1-u)\left(m-m_{3}-1\right)\right]\right\}
\end{aligned}
$$


For some researchers working the network security, they want to obtain the number of hub-vertices after getting the degree distribution $P(k)$, because they can protect it from interference by control some hub-vertices (Ref. [19], [25]) of the whole network. Next, we use the cumulative formula (Ref. [5]) here

$$
P_{\text {cum }}(k)=\frac{1}{n_{v}(t)} \sum_{k^{\prime} \gtrless k}\left|V\left(k^{\prime}, t\right)\right|
$$

Considering the integral property and continuity

$$
P_{\text {cum }}(k)=\frac{1}{n_{v}(t)} \sum_{k^{\prime} 2 k}\left|V\left(k^{\prime}, t\right)\right| \approx-\int_{k}^{n,(t)-1} P(k) d k
$$

Our model $N(t)$ in three cases, respectively, holds

$$
P_{\text {cum }}(k) \approx-\int_{k}^{n(t)-1} P(k) d k \approx l_{2}^{1 / Q_{2},} k^{-1 / Q_{2},}
$$

with $j=1,2,3$. By the analysts above, our model $N(t)$ is a scale-free model, since $2<1+1 / Q_{3 . j}<3$ by tuning suitably parameters $a, \alpha, \beta$ and $\mu$ with $j=1,2,3$.

\section{Summary}

According to a mass of research results about complex systems and networks at present, we firstly set up the general partial differential equation with 5 characteristic functions and achieve its solutions by abstract numerical analysis under a special initial condition. Secondly, considering the boundary of current research and the real-life background meanings, we establish one tuned parameters re-communication network model that has scale-free property. In this dynamical evolution process, we apply the degree-preferential-attachment mechanism (Ref. [24]) to make the model be scale-free and connect one part new adding vertices with edges to form masses of higher dense triangles for letting it be small-world. At the same time, the procedure of creating our models is so easy to do and achieve that we can build network models with the realistic topological structure, then explain and estimate its characters by simulating complex networks growing process. Our model may provide a useful tool to investigate the influence of the clustering coefficient or average path length in different dynamics processes taking place on networks. In addition, we propose some meaningful problems as the research in the future (Ref. [16], [26,] [27], [28]).

Problem 1. Reasonable proportion. In a growing network, the power-law parameter $\gamma$ will suffer these influence from the variational ratio between the number of isolate adding vertices and the number of simple graphs. Our models is a good case in point. This problem finding a golden ratio in complex systems will be discussed and debated for a long time in the future.

Problem 2. The diversity of adding graphs. In the dynamical evolution process of most real-life networks, not only isolate vertices are added, but also some simple graphs are entered. Themselves attachment rules are yet largely identical but with minor differences. This problem hunting a inner law in this randomness for describing dynamics processes of networks is on the way. The conclusion goes here.

\section{Acknowledgment}

We are grateful to the anonymous referees for their valuable and help comments which lead to the improvement of the paper. The third author, Bing Yao, thanks the National Natural Science Foundation of China under grants No. 61163054, No. 61363060 and No. 61662066. The fourth author, Ming Yao, thanks The Special Funds of Finance Department of Gansu Province of China under grant No. 2014-63. 


\section{References}

[1] J. A. Bondy and U. S. R. Murty. Graph Theory with Application. The MaCmillan Press Itd, London and Basingstoke, New York, 1976.

[2] D. J. Watts and S. H. Strogatz. Collective dynamics of small-world networks, Nature 393(1998)

[3] A. -L. Barabl'\{a\}si, R. Albert, H. Jeong. Mean-field theory for scale-free random networks. Physica A 272 (1999) 173-187.

[4] D. J. Watts. Small Worlds: The Dynamics of Networks between Order and Randomness. Princeton University Press, Princeton, NJ, 1999.

[5] M. E. J. Newman. The structure and function of complex networks. J. SIAM Review 45(2003)167-256.

[6] Z. Z. Zhang, S. Zhou, L. Fang, J. Guan. Y. Zhang. Maximal planar scale-free Sierpinski networks with small-world effect and power law strength-degree correlation. Europhys, Lett. 79 (2007) 38007.

[7] S. C. Chang, L. C. Chen, W. S. Yang. Spanning trees on the Sierpinskigasket. J. Stat. Phys. 126 (2007) 649-667.

[8] Z. Z. Zhang, S. Zhou. Collerations in random Apollonian networks. J. Physica A 380(2007)621-628.

[9] F. Comellas. Z. Zhang, L. Chen. Self-similar non-clustered planar graphs as models for complex networks. J. Phys. A: Math. Theor. 42 (2009) 045103.

[10] A. Miralles, F. Comellas, L. C. Chen, Z. Z. Zhang. Planar unclustered scale-free graphs as models for technological and biological networks. J. Phys. 389 (2010) 1995-19964.

[11] E. Teufl, S. Wagner. Resistance scaling and the number of spanning trees in self-similar lattices. J. Stat. Phys. 142 (2011) 879-897.

[12] F. Comellas, A. Miralles, H. X. Liu, Z. Z. Zhang. The number of spannings trees of an infinite family of outerplannar, small-world and self-similar graphs. J. Physica A 392 (2013) 2803-2806.

[13] Z. Z. Zhang, B. Wu, F. Comellas. The number of spanning trees in Apollonian networks. J. Discrete3 Applied Mathematics. 169 (2014) 206-213.

[14] G. Yan, G. Tsekenis, B. Barzel, J. JSlotine, Y. Y. Liu, A. L. Barabasi. Spectrum of controlling and observing complex networks. J. Nature Physics. 3422 (2015) 779-786.

[15] Erdos P, Renyi A. On random graphs. Publ. Math. , 1959, 6: 290-297

[16] B. Yao, M. Yao, X, E, Chen, X. Liu, W. J. Zhang, Research on Edge-Growing models related with scale-free small-world networks, J, Applied Mechanics and Materials, Volumes 513-517, 2014

[17] C. M. Song, T. Koren, P. Wang, A. L. Barabasi, Modelling the scaling properties of human mobility,J. Nature Physics. 1760 (2010) 1-6

[18] H. G. Liu, S. H. Cai, Y. Q. Zhang. The research on the scale-free networks model, J. College Physics, 2008 (4)

[19] G. Yan, G. Tsekenis, B. Barzel, J. JSlotine, Y. Y. Liu, A. L. Barabasi, Spectrum of controlling and observing complex networks, J. Nature Physics. 3422 (2015) 779-786

[20] Charo. I. Del. Genio, Thilo. Gross, Kevin. E. Bassler, All scale-free network are sparse, J. Physical review letters. 178701 (2011) 1-4

[21] Q. H. Chen, D. H. Shi. the modeling of scale-free networks, J. Physica A,2004, 335:240-248 
[22] H. Z. Liang, H. X. Yao, X. B. Zhang. Characters Analysis for a Class of Scale-Free Networks, J. Complex Systems and Complexity Science, 2005 (3)

[23] X. L. Jia, S. H. Cai, F. R. Zhang. A Dynamic Scale-free Network Model, J. Journal of Sichuan Normal University (Natural Science), 2009 (6)

[24] P. L. Krapivsky, S. Redner, F. Leyvraz, Connectivity of growing random network, J. Phys. Rev. Lett. 85,5901(2000)

[25] L. Wang, G. Z. Dai. On Degree Distribution of Complex Network, J. Journal of Northwestern Polytechnical University, 2006 (4)

[26] B. Yao, X. Liu, W. J. Zhang, X. E. Chen, X. M. Zhang, M.Yao, Z. X. Zhang. Applying Graph Theory To The Internet of Things. 2013 IEEE International Conference on High Perfermance Computing and Communication and 2013 IEEE International Conference on Embedded and Ubiquiyous Computing, 2354-2361

[27] B. Yao, M. Yao, X. E. Chen, X. Liu, W. J. Zhang. Research on Edge-Growing Models Related with Scale-Free Small-World Networks. Applied Meachanics and Materials,Vol. 513-517,2013,pp 2444-2448

[28] B.Yao, C. Yang, M. Yao, H. Y. Wang, X. E. Chen, X. M. Zhang, M. G. Li. Graphs As Models of Scale-free Networks. Applied Mechanics and Materials, Vol.380-384(2013)pp2034-2037. DOI: 10.4028/www.scientific.net/AMM.380-384.2034

[29] F. Q. Gu, S. H. Fan. The two-way evolution of a BA scale-free network, J. Journal of Jinan University (Natural Science), 2013 (5)

[30] A. Wu, X. S. Liu, D. Liu, C. S. Zuo. Group Growing Based Small-World Scale-Free Network Model, J. Journal of Computer Science, 2005 (7)

[31] Z. Z. Zhang, L. L. Rong, T. Zhou. An Evolving Model for Scale-free Collaboration Networks, J. System Engineering-Theory Practice, 2005 (25)

[32] Z. Z. Zhang, B. Wu, Y. Lin. Counting spanning trees in a small-world Farey graph, J. Physica A, 391(2012) 3342-3349

[33] Z. Z. Zhang, S. G. Zhou, Z. Su, T. Zhou, J. H. Guan. Random Sierpinski network with scale-free small-world and modular structure, J. Nature 2008 